\title{
Dezentral steuern, zentral kommunizieren - Ein Steuerungskonzept für wandelbare Materialfluss- systeme
}

\author{
DR.-ING. MICHAEL WILKE \\ LEHRSTUHL FÜR FÖRDERTECHNIK MATERIALFLUSS LOGISTIK, TU MÜNCHEN
}

Materialflusssysteme für kundenindividuelle beziehungsweise dynamische Produktionen werden heutzutage noch überwiegend mit manuellen Fördermitteln realisiert. Doch neue Lösungskonzepte im Bereich der Materialfluss- und Gerätesteuerung wie die funktionsorientierte Modularisierung und intelligente Multiagentensysteme bieten die Möglichkeit, wandelbare und automatisierte Materialflusssysteme in dynamischen Produktionsstrukturen einzusetzen. Diese wandelbaren Materialflusssysteme müssen dabei die Fähigkeit besitzen, auf ungeplante und nicht vorgedachte Ereignisse selbstständig reagieren zu können.

\section{Einleitung}

Die Situation der Produktionsunternehmen ist von einer Verkürzung der Innovationszyklen, einer rasant ansteigenden Anzahl an Produktvarianten und einem beschleunigten technischen Fortschritt gekennzeichnet [Cox-98]. Daraus resultiert ein steigender Kosten- und Zeitdruck im Wettbewerb der Unternehmen. Oftmals kann nur derjenige Wettbewerber Vorteile am Markt erzielen, der in der Lage ist, sich schnell auf verändernde Marktbedingungen und wechselnde Kundenwünsche optimal und kosteneffizient anpassen zu können [Schuh-04]. Der Kunde wird vom Konsumenten immer mehr zum Mitgestalter und verlangt nach individualisierten Produkten, die den eigenen Bedürfnissen entsprechen [SFB582-04]. So kann über die letzten Jahre hinweg ein starker Anstieg der Variantenvielfalt in den verschiedensten Produktbereichen und Branchen beobachtet werden. Dieser Trend und die damit verbundene Kleinserien- und Einzelfertigung führt zu kleineren Losgrößen und einem höheren Transportaufkommen in der Produktion.

Die Erfüllung dieser Anforderungen erfordert einen grundlegenden Neuaufbau der Fabrikstrukturen [West-03, Wien-02, Wirt-01]. Es bedarf zum einen flexible Produktionsstrukturen und wandelbare Materialflusssysteme. Zum anderen gilt es, die Wirtschaftlichkeitspotenziale zu nutzen, die sich z.B. durch eine Automatisierung der Prozesse ergeben können, um die Kostenschere gegenüber einer Serienfertigung zu verkleinern. Dabei müssen diese zukünftigen Systeme neben kurzen Reaktionszeiten und einer sehr hohen Flexibilität in dem Aufbau und in den Abläufen auch die Fähigkeit besitzen, auf ungeplante, nicht vorgedachte Szenarien selbstständig reagieren zu können.

Gegenwärtige automatisierte Materialflusssysteme werden aber oft als starr und unflexibel angesehen. Zusätzlich ist ihre Komplexität bei aufwendigen Systemen schwer zu beherrschen. Dies führt zu hohen Engineeringkosten sowie langen Inbetrieb- und Umbauzeiten. So kommen heutzutage in dynamischen Produktionsstrukturen meist nur manuelle Fördersysteme zum Einsatz. Diese verfügen aber weder über die höchste Produktivität noch über eindeutig quantifizierbare Kundenvorteile wie Nullfehler-Strategie oder zeitoptimierte Anwendungen. Darüber hinaus verursachen manuelle Transporte nicht nur hohe Betriebs- und Personalkosten, sondern weisen auch ein großes Gefahren- und Fehlerpotenzial auf und führen mitunter zu Qualitätseinbußen beim Fördervorgang [Dull-99].

Somit gilt es, neue Automatisierungslösungen zu entwickeln, die es erlauben, automatisierte Materialflusssysteme kostengünstig und flexibel in dynamischen Produktionsstrukturen einzusetzen. Ein Lösungsansatz liegt hier in der Entwicklung von autonomen, dezentral gesteuerten Materialflussmodulen, die über standardisierte, leistungsfähige Schnittstellen auf der physischen und auf der informationstechnischen Ebene verfügen.

Die Leistungsfähigkeit und die Wandelbarkeit solch eines modularisierten Materialflusssystems hängen dabei von der Leistungsfähigkeit und der Flexibilität der einzelnen materialflusstechnischen Komponenten sowie dem Zusammenspiel dieser Komponenten ab. Neben physischen Prozessen wie der Lastübergabe steht hier vor allem die Steuerungs- und Kommunikationstechnik vor großen Herausforderungen. 


\section{Anforderungen an ein wandelbares Materialflusssystem}

Wandelbare Materialflusssysteme müssen in Ergänzung zur Flexibilität, die als Möglichkeit zur Veränderung in vorgehaltenen Dimensionen und Szenarios bezeichnet werden kann, noch zusätzlich die Anforderung erfüllen, auf ungeplante und nicht vorgedachte eintretende Ereignisse selbstständig reagieren zu können.

Übertragen auf den Bereich der Materialflusstechnik lässt sich diese Definition der Wandelbarkeit in die Forderungen nach Layoutflexibilität, Durchsatzflexibilität und Fördergutflexibilität spezifizieren.

Dabei definieren sich die Flexibilitätskriterien wie folgt [Hand-01, Gün-02]:

- Fördergutflexibilität

- $\quad$ Layoutflexibilität

- Durchsatzflexibilität

Diese drei Flexibilitätskriterien lassen sich zu einer operativen Flexibilität zusammenfassen. Mit dieser werden Schwankungen im laufenden Betrieb, wie z. B. Auftragsmengenschwankungen und geringfügige Änderungen innerhalb des vorgegebenen Produktspektrums gehandhabt.

Um zu gewährleisten, dass ein Materialflusssystem in der Lage ist, auf jedes ungeplante Ereignis bzw. Aufgabe reagieren zu können, müssten diese drei Anforderungen vollständig erfüllt sein. Ein wirtschaftlicher Betrieb und eine technische Umsetzung wären unter diesen Gesichtspunkten nicht bzw. kaum zu realisieren.

Damit jedoch ein Materialflusssystem so gestaltet werden kann, dass eine Reaktion auf ungeplante Ereignisse und trotzdem noch ein wirtschaftlicher Betrieb möglich ist, müssen zusätzlich folgende Leitmotive erfüllt werden:

- $\quad$ Erweiterungsfähigkeit

(Hinzufügen von systemeigenen Materialflussmitteln/Modulen)

- Integrationsfähigkeit

(Hinzufügen von systemverschiedenen Materialflusssmitteln/Modulen)

Mit den Eigenschaften Erweiterungsfähigkeit und Integrationsfähigkeit besitzt das Materialflusssystem die Fähigkeit, auf vorab nicht geplante Änderungen zu reagieren. Diese Art der Reaktionsfähigkeit wird als strategische Wandelbarkeit bezeichnet. Sie kommt zum Einsatz, wenn die operative Flexibilität eines Materialflusssystems nicht mehr ausreicht, um auf Veränderungen im Produktspektrum entsprechend zu reagieren, bzw. den Umstieg auf neue Produkte zu bewältigen.

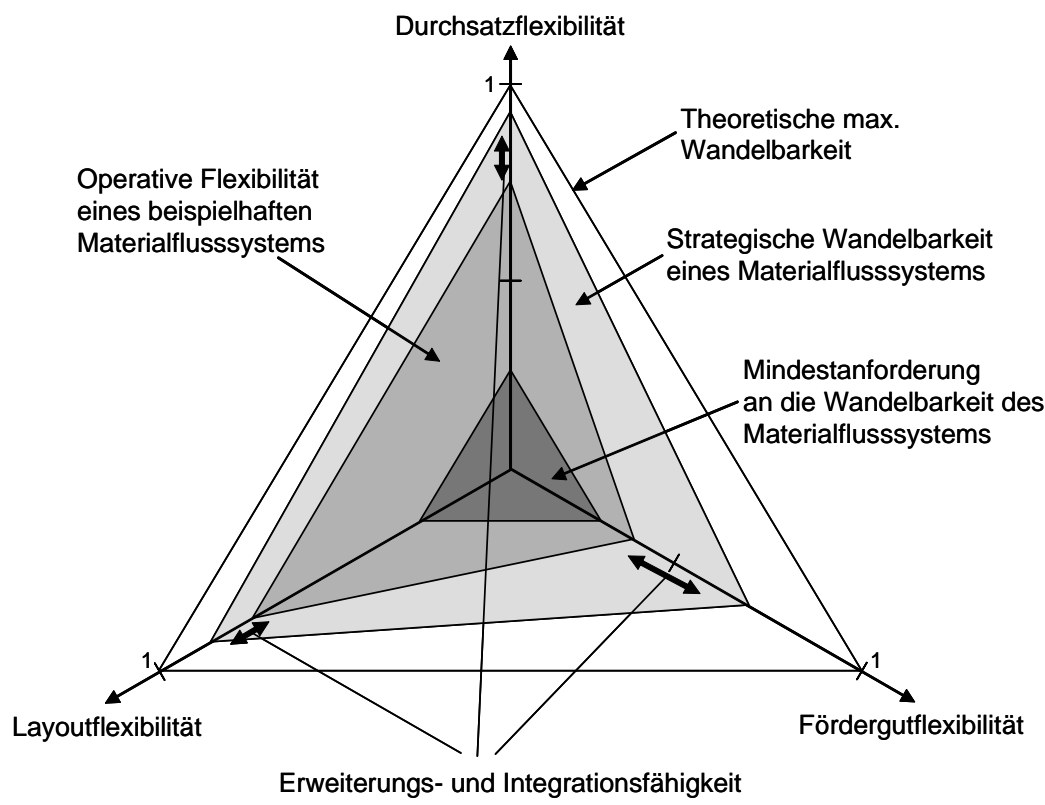

Abbildung 1: Beurteilung der Wandelbarkeit eines Materialflusssystems (Beispiel) 
Ob ein Materialflusssystem wandelbar ist, hängt von der Gestaltung seiner Systemkomponenten ab. Diese müssen in ihrer technischen Realisierung die Leitmotive Erweiterungs- und Integrationsfähigkeit aufweisen. Hierzu sind bei der Systemgestaltung neue Ansätze in der Modularisierung und Automatisierung nötig, wie sie im Folgenden beschrieben werden.

\section{Gestaltungsmethodik: Funktionsorientierte Modularisierung}

Ein wandelbares automatisiertes Materialflusssystem erfordert einen konsequenten, dezentralen Modularisierungsansatz, der nicht nur die mechanische Seite berücksichtigt, sondern auch auf energetischer und steuerungstechnischer Seite autonome und eigenintelligente Komponenten vorsieht. Als Gestaltungsmethode bietet sich eine funktionsorientierte Modularisierung in Verbindung mit einer mechatronischen Betrachtungsweise an. Bei dieser Methode wird das Materialflusssystem aus mechatronischen Modulen aufgebaut, deren Modulgrenzen entsprechend der Funktionalität gezogen und die nach dem Konzept der verteilten Automatisierung automatisiert sind. Ein Modul definiert sich nach der Funktion bzw. Aufgabe, die es erfüllt, wie z. B. das Transportieren. Alle Teilnehmer des Materialflusssystems wie Krane, Katzen bzw. EHBFahrzeuge, Weichen und Übergabeplätze stellen autonome mechatronische Module dar, deren gesamte Funktionalität in Form von Mechanik, Energie und Steuerung zu einer mechatronischen Funktionseinheit vereinigt wird. Die Definition der Funktionalität, der Leistungsfähigkeit und der Geometrie des Moduls sowie dessen Schnittstellen werden in speziellen XMLModulbeschreibungen festgehalten [Wil-06].

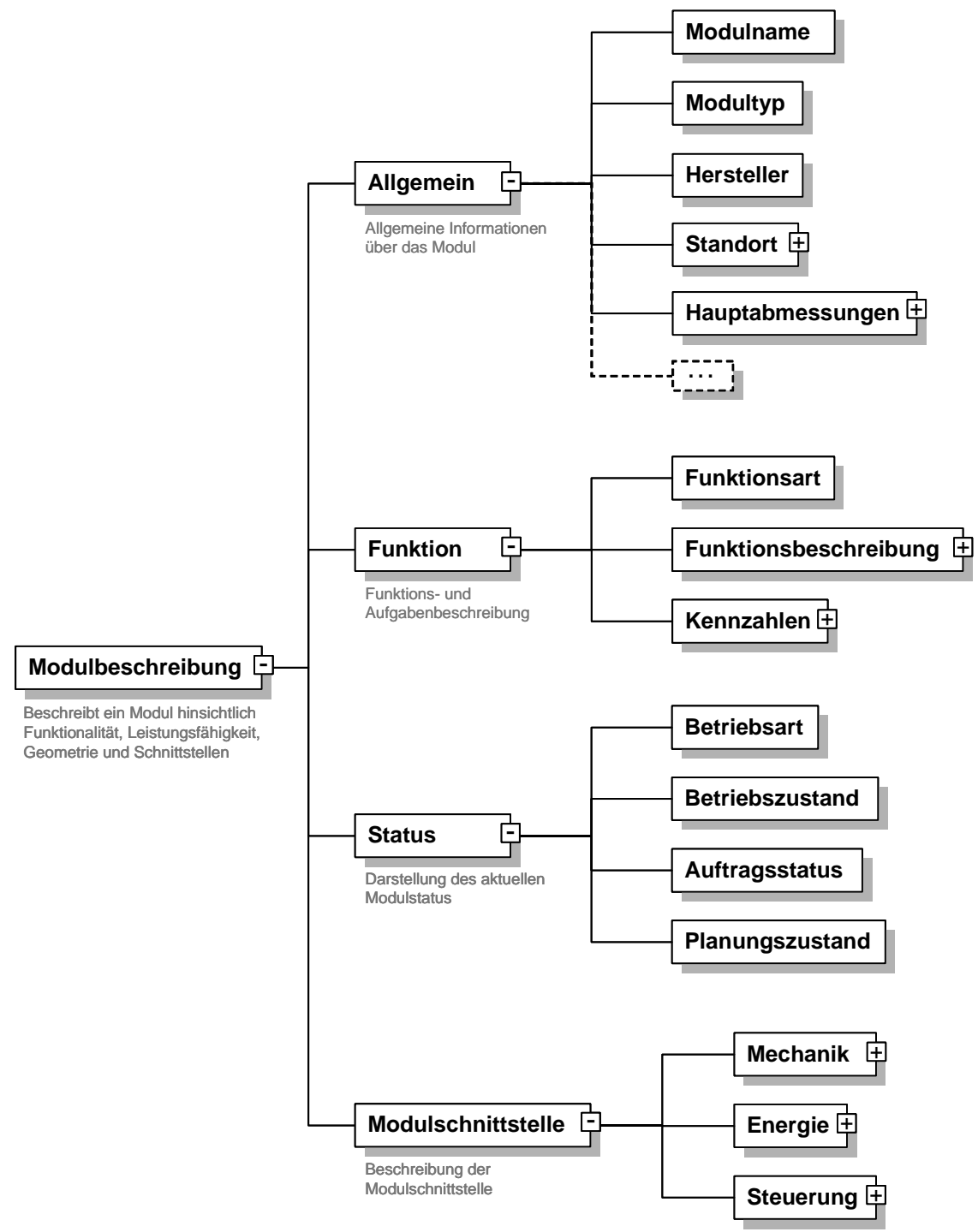

Abbildung 2: Schematischer Aufbau der XML-Modulbeschreibung 


\section{Kommunikationskonzept}

Damit in einem wandelbaren modularisierten Materialflusssystem materialflusstechnische Module verschiedener Hersteller ohne großen Engineeringaufwand miteinander gekoppelt, ausgetauscht bzw. neu eingebunden werden können, müssen die Module über eine einheitliche, kompatible und leistungsfähige Kommunikationsschnittstelle verfügen. Diese Schnittstelle muss so definiert sein, dass sie auch den Anforderungen zukünftiger neuer Materialfluss- und Produktionsmodule entspricht, ohne dass eine explizite Vorplanung nötig ist. Es muss sowohl eine horizontale Kommunikation zwischen den Modulen als auch eine vertikale Kommunikation zu übergeordneten PPS und ERP-Systemen möglich sein. Analog zu den beschriebenen Anforderungen an die Wandelbarkeit eines Materialflusssystems stehen bei der Kommunikation die Eigenschaften Erweiterungs- und Integrationsfähigkeit im Vordergrund.

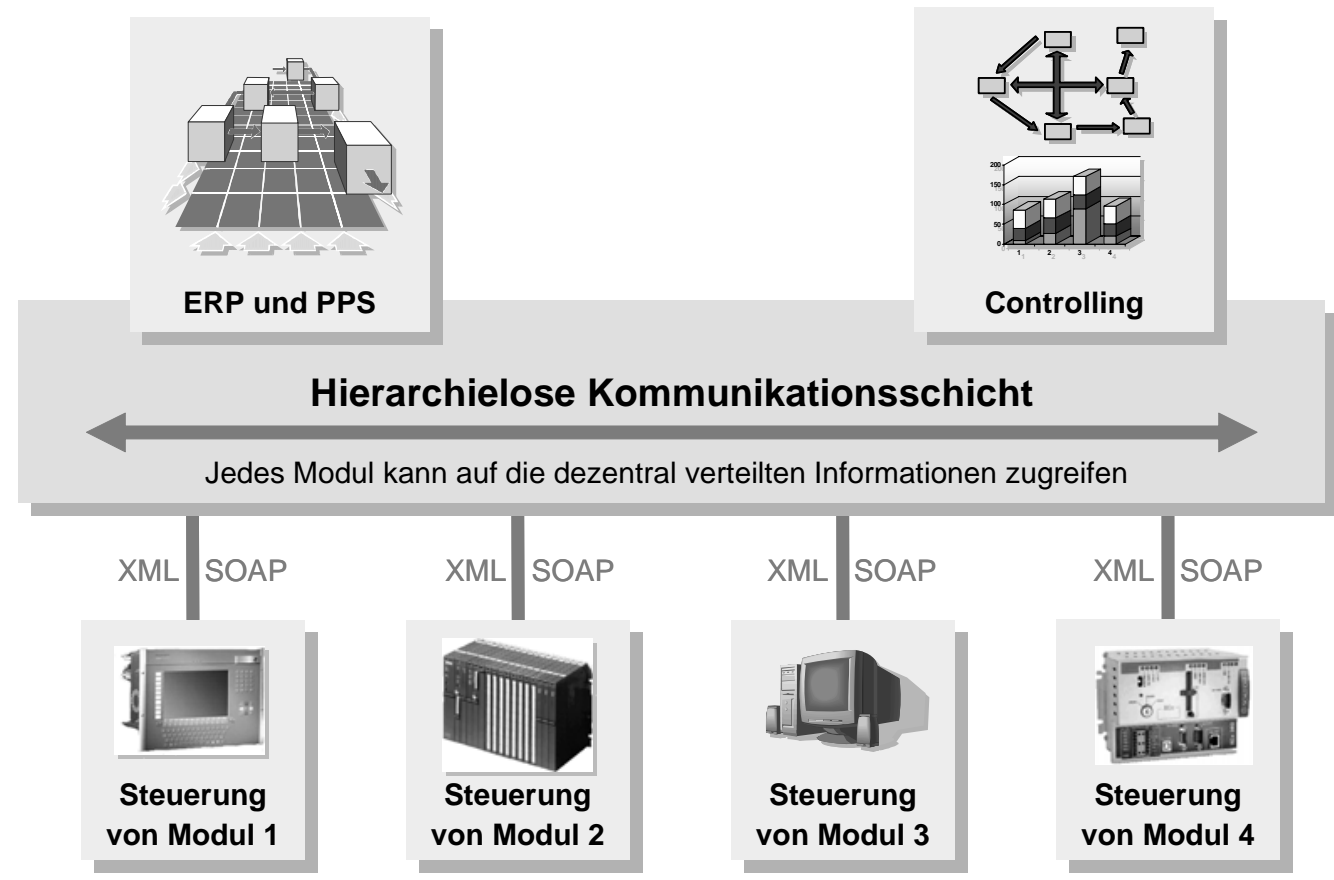

\section{Abbildung 3:Kommunikationsarchitektur bei einer funktionsorientierten Modularisierung}

Einen geeigneten Lösungsansatz bietet hier die Datensprache XML (Extensible Markup Language), die aus dem IT-Bereich stammt. XML ist eine Metasprache, die Regeln für die Erstellung von Dateistrukturen auf Basis von Textformaten beschreibt. Sie erlaubt eine Definition der logischen Bedeutung von Daten, Informationen und Texten. Neben der eigentlichen Information werden auch gleichzeitig die Datenbezeichnung und das Datenformat übermittelt. Mit Hilfe eines einfachen Web-Browsers lassen sich die XML-kodierten Daten einfach und optisch aufbereitet lesen. Ein Datenaustausch auf Basis von XML schafft damit die Voraussetzungen für eine erweiterungsfähige offene Kommunikation in einem wandelbaren Materialflusssystem.

Das Kommunikationskonzept verfolgt den Grundsatz, dass die Module dezentral gesteuert werden, aber über eine gemeinsame hierarchielose Kommunikationsschicht miteinander verbunden sind. Die Daten werden dezentral erzeugt, lassen sich immer einem Modul zuordnen und sind aber jederzeit für alle anderen Module zugänglich.

\section{Fahrzeugsteuerungskonzept}

Aufbauend auf den Wandelbarkeitsanforderungen und unter der Berücksichtung des Konzeptes der funktionsorientierten Modularisierung sowie einem Kommunikationskonzept auf Basis von XML, entstand am Lehrstuhl fml, TU München, folgendes Fahrzeugsteuerungskonzept für ein wandelbares Materialflusssystem [Wil-06]. Die Funktionsweise des Konzeptes lässt sich an einem Analogiebeispiel veranschaulichen. 
Das Straßennetz stellt mit den Autofahrern ein wandelbares Materialflusssystem dar. Jeder Autofahrer kann jeden Ort unabhängig von den anderen Fahrern erreichen und dabei die unterschiedlichsten Dinge transportieren.

Dazu plant der Autofahrer seine Route anhand einer Straßenkarte und orientiert sich unterwegs an den Ortsschildern bzw. Wegweisern. Dabei spielt es für ihn im Gegensatz zu einem starr automatisierten System keine Rolle, ob er an zwei oder mehreren Abzweigungen vorbeikommt oder sogar ungeplante Umleitungen fahren muss. Seine Wandelbarkeit beruht auf dem Auswerten einer Straßenkarte und der Orientierung anhand von Straßenschildern.

Das entwickelte Fahrzeugsteuerungskonzept adaptiert dieses Verhalten mit Hilfe von einfachen Regeln. Dazu benötigen die Fahrzeuge eine Art Straßenkarte. Diese ist eine Matrix, in der die Informationen abgelegt sind, welche Orte (z.B. Übergabestellen, Weichen, etc.) über welche Art von Wegen miteinander verbunden sind. Diese Matrix wird als Waypointmatrix bezeichnet. Sie stellt quasi das informationstechnische Abbild des Materialflusssystems dar und übernimmt die Funktion der oben beschriebenen gemeinsamen Kommunikationsschicht. Sie spiegelt das Layout, die Eigenschaften (z.B. Länge der Strecken, Streckenkapazitäten) und den aktuellen Zustand des Materialflusssystems (z.B. Reservierungen, Positionen der Fahrzeuge, etc.) wider und zeigt so nicht nur den statischen, sondern auch den dynamischen Zustand des Systems an.

Die Daten in der Waypointmatrix, kodiert in XML, werden von allen Modulen, die an Transportvorgängen beteiligt sind (Fahrzeuge, Weichen, Hängekrane etc.), benötigt. Dabei werden die Informationen nicht nur ausgelesen, sondern auch von den Modulen verändert. Ein Fahrzeug berechnet dezentral und vollkommen autonom seinen Fahrweg. Anschließend wird dieser in Form von Reservierungen in die Matrix eingetragen. Das ermöglicht den Fahrzeugen, die Positionen und Fahrwege der anderen Fahrzeuge bei der eigenen Wegplanung zu berücksichtigen, ohne mit diesen direkt kommunizieren zu müssen. Durch die Vergabe eines umlaufenden Tokens wird gewährleistet, dass jeder Teilnehmer den aktuellen Zustand der Waypointmatrix erhält. Nur das Modul, das den Token besitzt, darf die Matrix verändern [Wil-06]. So werden von vornherein konkurrierende Reservierungen vermieden. Aber nicht nur die Fahrzeuge holen sich die Informationen aus der Matrix. Ebenso erfahren die Weichenmodule, die in der Matrix einen Waypoint darstellen, aus den Reservierungen, wie sie sich zu stellen haben.

Neben der Waypointmatrix als Straßenkarte benötigen die Transportfahrzeuge des Materialflusssystems Straßenschilder, an denen sie sich unterwegs orientieren können. Technisch lassen sich diese Straßenschilder mit Transpondern (RFID-Technologie) realisieren. Die Transponder verfügen über eine eindeutige Kennung und sind neben der Fahrbahn angebracht. Sie markieren Übergabestellen und Verzweigungen und stellen damit die so genannten Waypoints in der Waypointmatrix dar. Die Fahrzeuge lesen im Vorbeifahren die Transponder aus, um zu erfahren, wo sie sich befinden.

\section{Vorteile}

Dieses Konzept bietet eine sehr hohe Layoutflexibilität. Informationen über den physischen Streckenverlauf sind an nur einem Ort, der Waypointmatrix, steuerungstechnisch hinterlegt. Dort lassen sich Veränderungen in der Topologie schnell und einfach vornehmen. Selbst bei nicht vorgeplanten Veränderungen des Wegsystems müssen die Fahrzeugsteuerungen nicht umprogrammiert werden, da die Transportfahrzeuge alle benötigten Informationen für eine dezentrale Wegplanung aus der Waypointmatrix beziehen.

Ebenso zeichnet sich dieses Konzept durch eine hohe Ausfallsicherheit aus. Fallen aufgrund von Störungen Streckenabschnitte aus, sind die Fahrzeuge in der Lage, sich selbstständig Alternativrouten zu suchen, ohne dass diese vorher festgelegt werden müssen.

Ferner erlaubt das entwickelte Konzept eine hohe Durchsatzflexibilität. Das Hinzufügen von Fahrzeugen ist ohne Softwareänderungen jederzeit möglich, da die Fahrzeuge nicht direkt miteinander kommunizieren, sondern über die Reservierungseinträge in der Waypointmatrix erfahren, ob Strecken frei oder von anderen Teilnehmern belegt sind. Dies erlaubt ebenso ein Befahren der Wege in beide Fahrtrichtungen, um z. B. durch eine kurze Rückwärtsfahrt den schnellsten Weg zu nehmen. Die Fahrzeuge reservieren nur eine gewünschte Fahrtrichtung, ohne die komplette Strecke für andere zu sperren. Nachfolgende Fahrzeuge können so die Teilstrecken in gleicher Fahrtrichtung mitbenutzen.

Die Wegplanung wird grundsätzlich dezentral von der Fahrzeugsteuerung übernommen. Dabei ist es sogar möglich, dass Fahrzeuge mit unterschiedlichen Strategien und Fähigkeiten nebeneinander in dem gleichen System operieren können. Dies wird zum einen durch die funktionsorientierte Modularisierung und zum anderen durch die Waypointmatrix ermöglicht. 
Dieses Steuerungskonzept für dezentral gesteuerte autonome Fördermittel ist erweiterungs- und integrationsfähig und erfüllt damit die wichtigen Leitmotive für ein wandelbares automatisiertes Materialflusssystem. Es ist sowohl für Elektohängekran-/Hängebahnsysteme als auch für Fahrerlose Transportsysteme anwendbar.

Durch die strikte funktionsorientierte Modularisierung und die klar gezogenen Systemgrenzen mit ihren standardisierten mechatronischen Schnittstellen werden außerdem unerwünschte redundante Steuerungsvorgänge bzw. Datenhaltung vermieden.

In diesem Konzept wird die Funktionalität eines Materialflussrechners auf das Vergeben von Transportaufträgen beschränkt. Aufgaben wie die Wegplanung und Stauverhinderung, die üblicherweise nach dem VDMA-Ebenenmodell [VDMA-15276] von der Subsystemsteuerung zentral gelöst werden, werden hier dezentral von den Fahrzeugen übernommen.

\section{Zusammenfassung}

Heutzutage werden Materialflusssysteme für kundenindividuelle bzw. dynamische Produktionen noch überwiegend mit manuellen Fördermitteln realisiert. Doch neue Lösungskonzepte im Bereich der Materialfluss- und Gerätesteuerung wie die funktionsorientierte Modularisierung [Gün-05, Wil-06] und intelligente Multiagentensysteme [Homp-05] bieten in den nächsten Jahren die Möglichkeit, wandelbare und automatisierte Materialflusssysteme in dynamischen Produktionslandschaften einzusetzen.

Der Einsatz von autonomen, dezentral gesteuerten Modulen mit standardisierten Schnittstellen im Bereich des physikalischen und des informationstechnischen Materialflusses ist die Grundlage für die Realisierung eines kostengünstigen, effektiven, zuverlässigen und wandelbaren Gesamtsystems. Die Zeitanteile bei der Inbetriebnahme und vor allem beim Umbau lassen sich damit deutlich reduzieren.

Die Zusammenarbeit der Module wird durch eine gemeinsame, hierarchielose Kommunikationsschicht auf Basis von XML erreicht, die einen offenen und erweiterungsfähigen Datenaustausch sowohl horizontal zwischen den Materialflussmodulen als auch vertikal von der Leitebene bis hinunter auf die Geräteebene ermöglicht.

Prototypenhafte Umsetzungen und materialflusstechnische Simulationen zeigen das große Potential hinsichtlich Wandelbarkeit und Leistungsfähigkeit solcher Systeme auf. Erste Lösungsansätze hierfür sind am Lehrstuhl für Fördertechnik Materialfluss Logistik der TU München erarbeitet worden.

\section{Literatur}

[Cox-98]

[Dull-99]

[Gün-02]

[Gün-05]

[Hand-01]

[Homp-05]
Cox, M.; Alm, R.: The right stuff: America's move to mass customization, Federal Reserve, Bank of Dallas, 1998 Annual Report, S. 3-26,1998.

Dullinger, K. H.: Das Zauberwort heißt Flexibilität - Wie sich Logistikanlagen von vornherein flexibel für zukünftige Anforderungen anlegen lassen. IN: Materialfluss 30(1999)4, S. 15-18.

Günthner, W.A.; Heinecker, M.; Wilke, M.: Materialflusssysteme für wandelbare Fabrikstrukturen. IN Industrie Management 18(2002)5, S. 8-10.

Günthner, W.A.; Wilke, M.: Mit neuen Lösungen $\mathrm{zu}$ wandelbaren Materialflusssystemen. IN: Intelligenter produzieren (2005)4, S. 5-6.

Handrich, W.: Flexible, flurfreie Materialflusstechnik für dynamische Produktionsstrukturen (Dissertation). München: Utz Verlag, München, 2001.

ten Hompel, M; Lammers, W.: Das Internet der Dinge - Die RFIDTechnologie ermöglicht die Selbstorganisation logistischer Prozesse. Ein grundlegender Wandel für den automatisierten Materialfluss

IN: Logistik inside (2004), Sonderheft Who is Who der Logistik 2005, S.118-120. 
[Schuh-04]

[SFB582-04]

[VDMA-15276]

[West-03]

[Wien-02]

[Wil-06]

[Wirt-01]

[Zäh-03]
Schuh, G.; Harre, J.; Gottschalk, S.; Kampker, A.: Design for Changeability (DFC) - Das richtige Maß an Wandlungsfähigkeit finden, Ergebnisse des EU-Verbundforschungsprojektes „Modular Plant Architecture“. IN: wt Werkstattstechnik online 94(2004)4, S. 100-106.

SFB 582: Marktnahe Produktion individualisierter Produkte, http:/www.sfb582.de, TU München, 2004.

Verband Deutscher Maschinen- und Anlagenbau (VDMA): VDMAEinheitsblatt 15276: Datenschnittstellen in Materialflusssystemen. Berlin: Beuth, 1994.

Westkämper, E.; Kirchner, S.; Winkler, R.: Unternehmensstudie zur Wandlungsfähigkeit von Unternehmen. IN: wt Werkstattstechnik online 93(2003)4, S. 245-260.

Wiendahl, H-.P.: $\quad$ Wandlungsfähigkeit $\quad-\quad$ Schlüsselbegriff der zukunftsfähigen Fabrik. IN: wt Werkstattstechnik online Jahrgang 92(2002)4, S. 122-127.

Wilke, M.: Wandelbare automatisierte Materialflusssysteme für dynamische Produktionsstrukturen (Dissertation). München: Utz Verlag, 2006.

Wirth, S.; Baumann, A.: Wertschöpfung durch vernetzte Kompetenz Schlanke Kompetenzkooperation. München: Huss, 2001.

Zäh, M.; Ulrich, C.; Wagner, W.; Wilke, M.: Reaktionsszenario Minifabrik, IN: wt Werkstattstechnik online 93(2003)9, S. 646-650 